\title{
Tailings Filtration Using Viper Filtration Technology-a Case Study
}

\author{
Oliver Whatnall ${ }^{1} \cdot$ Kevin Barber $^{1} \cdot$ Peter Robinson $^{2}$ \\ Received: 28 September 2020 / Accepted: 5 January 2021 / Published online: 25 January 2021 \\ (C) The Author(s) 2021
}

\begin{abstract}
Investigation and uptake of filtered tailings continues to grow throughout the globe. This is driven by a wide range of site-specific considerations, which include such factors as tailings characteristics (e.g., amenability to filtration), production rates, climate, water availability, cost drivers, environmental requirements, and social factors. Despite the aforementioned technological growth, the currently available filtration technology is not able to meet the needs of many operations and projects that would otherwise adopt the technology. Experience with large-scale industrial filtration shows that vacuum belt filter systems meet the needs of many modern users, exceptions being the inability to effectively dewater tailings at altitude and/or with a fine particle size distribution: a potential fatal flaw. This paper presents a case study on the utilization of the patented Viper Filtration technology on gold tailings to overcome this challenge and shares the resultant full-scale plant design, highlighting the features designed to overcome cost and scalability deterrents. This technology is a novel mechanical process which complements the vacuum pressure in dewatering the filter cake as it travels along the belt filter. This project commenced with a pilot testing program, which successfully met the objective to rigorously test, measure and record any performance improvements achieved when engaging the Viper technology. Of the two tailings products tested, gross improvements of $4.2 \% \mathrm{w} / \mathrm{w}$ and $5.7 \% \mathrm{w} / \mathrm{w}$ were achieved when compared to the conventional vacuum belt filter operation. This pilot testing facilitated measurement of operating and design data, which forms the basis of the full-scale system design and resultant equipment supply of three vibration roller assemblies for retro-fitting on the existing vacuum belt filter.
\end{abstract}

Keywords Tailings $\cdot$ Dewatering $\cdot$ Filtration $\cdot$ Dry-stack $\cdot$ Gold $\cdot$ Viper

\section{Introduction}

Experience with large-scale industrial filtration shows that vacuum belt filter systems meet the needs of many modern users, exceptions being the inability to effectively dewater tailings at altitude and/or with a fine particle size distribution: a potential fatal flaw. This paper presents a case study on the utilization of the patented Viper Filtration technology on gold tailings to overcome this challenge and shares the resultant full-scale plant design, highlighting the features designed to overcome cost and scalability deterrents. This technology is a novel mechanical process which complements the vacuum pressure in dewatering the filter cake as it travels along the belt filter.

Oliver Whatnall

owhatnall@jord.com.au

1 Jord International Pty Ltd, Shortland, NSW, Australia

2 The University of Newcastle, Callaghan, NSW, Australia

\subsection{Filtered Tailings}

Investigation and uptake of filtered tailings continues to grow throughout the globe. This is driven by a wide range of sitespecific considerations, which include such factors as tailings characteristics (e.g., amenability to filtration), production rates, climate, water availability, cost drivers, environmental requirements, and social factors. Despite the aforementioned technological growth, the currently available filtration technology is not able to economically meet the needs of many operations and projects that would otherwise adopt the technology. Currently, for tailings to reach the desired moisture content for dry stacking, the majority of processes require pressure filtration, with significant upfront and ongoing costs associated with maintenance. In line with this, the 2017 Study of Tailings Management Technologies report commissioned by the Mine Environment Neutral Drainage (MEND) Program Secretariat identified "cost and scalability of filtered tailings is one of the deterrents for mining companies." Given the comparative lower costs associated with low pressure Viper vacuum belt filter, this has the potential to alleviate dependency on high pressure applications and make efficient 
dewatering and handling of tailings a more economic and sustainable solution.

\subsection{Viper Filtration Technology}

A recently commercialized, patented technology (patent 20200166274) utilizes a series of vibration roller assemblies which are coupled to the top side of a vacuum belt filter. These rollers, incorporated with other Viper Filtration components, complement the vacuum pressure in dewatering the filter cake as it travels along the belt filter. Given the cost and scalability deterrents frequently encountered with pressure filtration of tailings, this technology has the potential to overcome these issues, by allowing Viper vacuum belt filter technology to be used in some applications.

\subsection{Gold Mine and Processing Plant}

This paper presents a case study for an underground gold mine. The mine produces $500,000 \mathrm{oz}(14,174 \mathrm{~kg}$ equivalent) gold per annum via a conventional carbon-in-pulp (CIP) processing plant. This processing plant produces slurry tailings of which approximately 100 tph is filtered to a low moisture content and then trucked to the mine to re-pulp as paste backfill.

In 2017, a conventional vacuum belt filter with a filtration area of $158 \mathrm{~m}^{2}$ was installed to wash (i.e., remove cyanide) and dewater the tailings for paste backfill. Since that time, the specification for paste backfill has been for a finer particle size distribution (PSD) of solids, which could not be effectively dewatered at the required rate from the existing vacuum belt filter.

\subsection{Pilot Plant Testing}

This project commenced with a pilot testing program, which successfully met the objective to rigorously test, measure, and record any performance improvements achieved when engaging the Viper technology compared to the conventional vacuum belt filter. This pilot testing facilitated measurement of operating and design data, which forms the basis of the fullscale system design and resultant equipment supply of three vibration roller assemblies for retro-fitting on the existing vacuum belt filter.

\section{Process Summary}

\subsection{Original Design Basis}

A portion of the slurry tailings from the process plant are deslimed and thickened to meet the desired specification for paste backfill. The tailings are washed for residual cyanide removal and dewatered on the vacuum belt filter prior to being stockpiled as filter cake. To facilitate the design and supply of the $158 \mathrm{~m}^{2}$ vacuum belt filter, the original specification for paste backfill was to wash and dewater $131 \mathrm{tph}$ of solids to less than $17 \% \mathrm{w} / \mathrm{w}$ moisture with a P80 ranging from 150 to $190 \mu \mathrm{m}$, with $15-20 \%<$ $20 \mu \mathrm{m}$ and $7-10 \%<10 \mu \mathrm{m}$.

\subsection{Project Justification}

The tailings deslime and filtration circuit was commissioned in late 2017, meeting design expectations for feed to the underground paste fill plant. Since 2017, the specification for paste backfill has been revised to a finer PSD, of the order of $40 \%<20$ um which cannot be effectively dewatered at the required rate from the existing vacuum belt filter. Modifying the filter plant in order to achieve this paste specification at existing design throughput rates is the objective of the project.

\section{Pilot Plant Testing}

\subsection{Objective}

The objective of the pilot program was to rigorously test, measure, and record any process performance improvements achievable using the Viper Filtration technology compared to the pilot-scale conventional vacuum belt filter. The pilot-scale testing begins with the control to obtain the baseline before the technology is engaged.

Additionally, the pilot testing allows for measurement of operating and design data, which will form the basis of the full-scale Viper modules and retrofit design. The testing program considered two sample types, namely, CIP tails $\left(\mathrm{P}_{80} \sim 40 \mu \mathrm{m}\right)$ and tailings thickener underflow (TUF), a combination of CIP tails and slimes from the existing tailings deslime circuit $\left(\mathrm{P}_{80} \sim 30 \mu \mathrm{m}\right)$, both of which are deemed acceptable for feeding into the paste backfill plant. PSD information is shown in Fig. 1.

Specifically, the target process performance is consistent with achieving $<20 \%$ moisture content by weight $(\% \mathrm{w} / \mathrm{w})$ of the tailings filter cake, required to facilitate trucking, while maximizing the throughput rate.

\subsection{Equipment}

The pilot plant is installed and operated at The University of Newcastle in New South Wales, Australia. The base filter is a vacuum belt filter with $1.6 \mathrm{~m}^{2}$ of filtration area $(0.4 \mathrm{~m}$ width $\times$ $4.0 \mathrm{~m}$ length). Installed in series on the top side of the machine are three vibration roller assemblies, each bolted to the filter frame as shown in Fig. 2. The filtration plant also includes the 
Fig. 1 PSD charts comparing CIP and TUF tailings

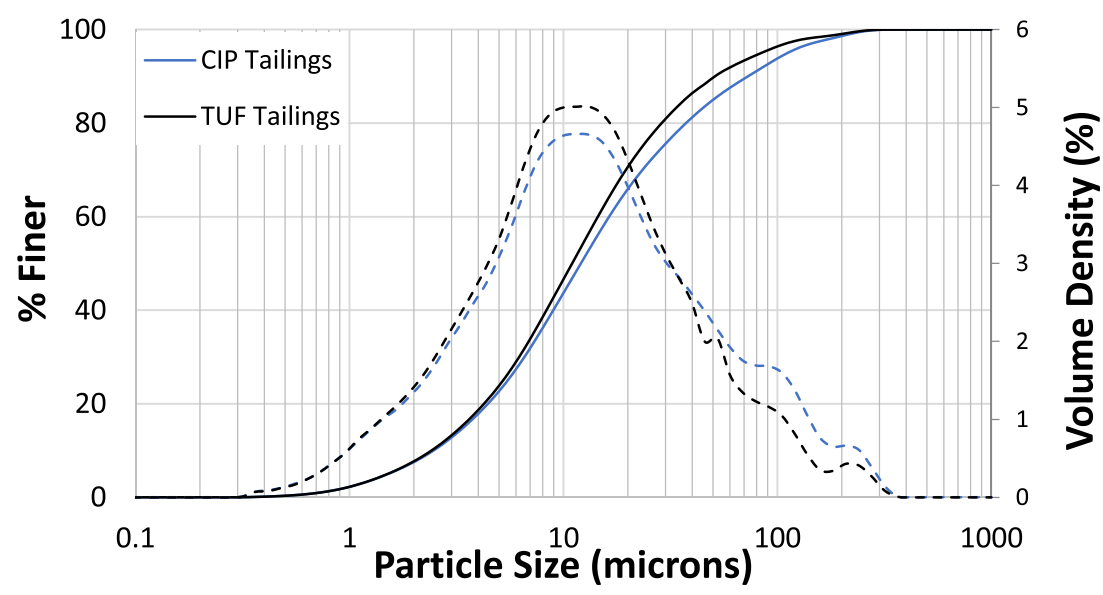

filter ancillaries - filtrate receiver vessel, filtrate pump, and liquid ring vacuum pump.

\subsection{Process Description}

The slurry tailings are pumped onto the filter at $40 \% \mathrm{w} / \mathrm{w}$ solids via the feedbox. The filter feed is continuously presented at the feed end of the machine and begins traveling along the length of the filter via the moving filter cloth and carrier belt (see Fig. 3).

Note: this feed solid content is lower than the design basis of $55 \% \mathrm{w} / \mathrm{w}$ solids, and this testing does not consider the cake washing step. Parallel bench scale vacuum belt filter testwork is used to inform the full-scale performance expectations and full-scale equipment design.

The pilot filter has distinct zones/areas:

i. Feed - the feed slurry is spread evenly across the width of the belt, by the overflowing weir (see Fig. 3), and is immediately exposed to the vacuum. For the given feed

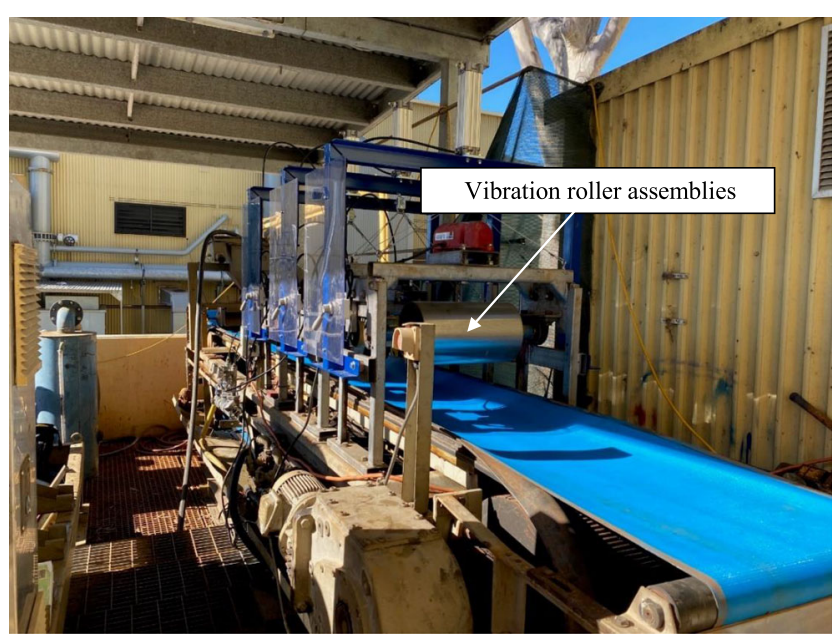

Fig. 2 Pilot vibration roller assemblies slurry, the cake thickness is set by the speed of the belt and the flowrate of the feed pump. The cake thickness was a maximum of $12 \mathrm{~mm}(\mathrm{~mm})$ and was consistently operating between 9 and $12 \mathrm{~mm}$.

ii. Form - the prepared slurry continues to be subjected to vacuum and results in filtrate being drawn from the slurry. The slurry is formed into a wet "cake", with or without surface water prior to the vibration roller assemblies.

iii. Mechanical dewatering - the cake travels under a series of three vibration rollers which provide consolidation, compaction, and vibration energy into the cake while under constant vacuum pressure. Each vibration roller assembly can be optimized by changing the roller weight,

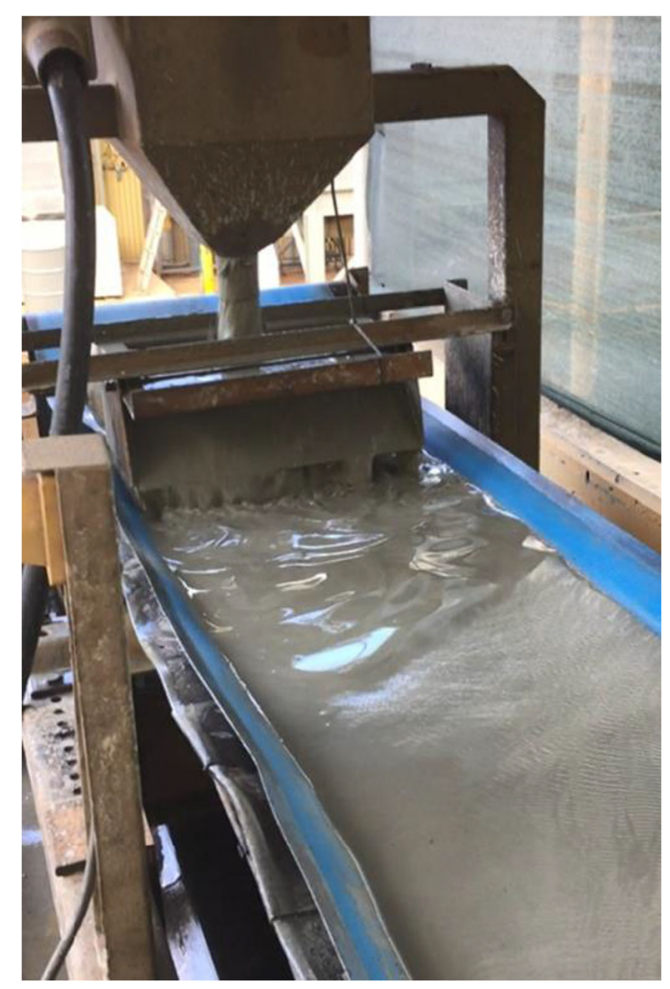

Fig. 3 Filter feedbox and overflowing weir distribution system 
Fig. 4 Filter cake discharge

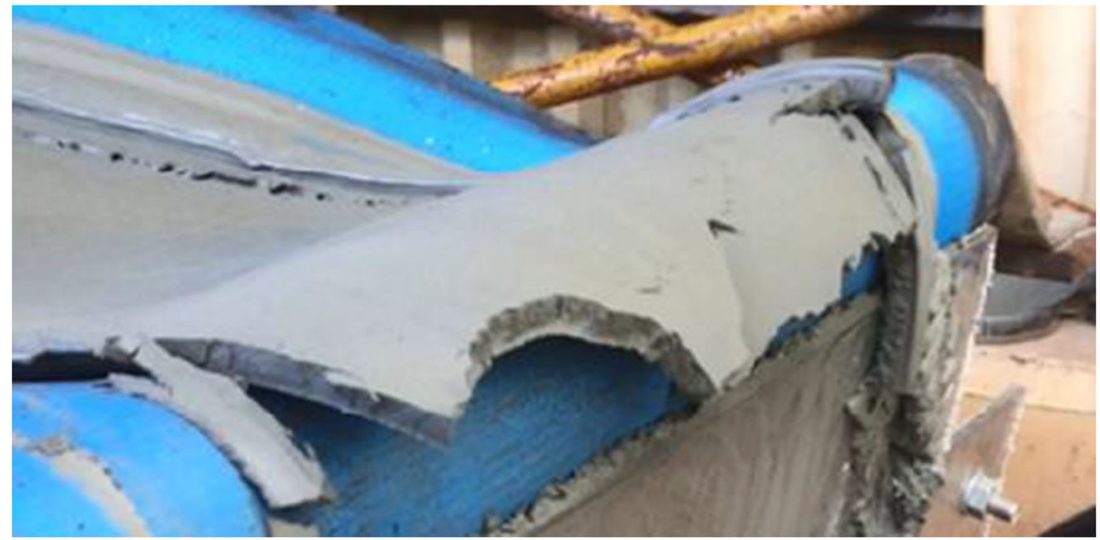

diameter, surface profile, vibrator type, and vibration intensity as well as flexibility to reposition the roller along the length of the filter. Post the third and final vibration roller, vacuum continues to be applied to the cake to allow further drying. This is the final stage of the filtration process.

iv. Cake discharge - following filtration, the filter cloth follows an alternate path and separates from the carrier belt. The filter cloth travels over the cracking roller and discharges the cake continuously (see Fig. 4).

v. Filtrate and vacuum system-all filtrate drawn from the slurry via the vacuum box is captured in the filtrate receiver vessels and is continuously pumped from the vessels by the filtrate pumps. Vacuum is provided by the liquid ring vacuum pump, which connects via the filtrate receivers through the vacuum hoses and vacuum box. The design vacuum suction pressure was -70 $\mathrm{kPa}$.

vi. Filter cloth wash - following cake discharge, the cloth is cleaned with water by spray bars to ensure process and cloth tracking performance.

vii. Filter cloth and belt return - both the filter cloth and carrier belt travel independently along the underside of the machine. Upon return to the feed end, both travel under the dam roller, which creates a seal prior to the feed preparation zone

\section{Results}

Results from the pilot program were measured and recorded through the course of processing CIP tailings and TUF tailings. Visual inspection recorded cleaner and more efficient cake discharge when operating the pilot plant with the vibration roller assemblies engaged compared to the conventional vacuum belt filter. Figure 5 shows the snapshot of the results for both sample types, with and without the vibration roller assemblies.

Across both tailings types tested, cake moistures $>20 \%$ w/ $\mathrm{w}$ were recorded when operating the pilot plant as a conventional vacuum belt filter. When operating the pilot plant with three vibration roller assemblies engaged, cake moisture contents $<20 \% \mathrm{w} / \mathrm{w}$ were measured for the CIP tailings for the range of cycle times between 142 and $119 \mathrm{~s}$ and for the TUF tailings with a cycle time of $119 \mathrm{~s}$.

When processing CIP tailings, on average, a $4.2 \% \mathrm{w} / \mathrm{w}$ moisture content reduction was recorded when operating the pilot plant with the Viper Filtration system engaged compared to the conventional vacuum belt filter. When processing TUF tailings, on average, a $5.7 \% \mathrm{w} / \mathrm{w}$ moisture content reduction was recorded when operating the pilot plant with the Viper Filtration system engaged compared to the conventional vacuum belt filter. Further work is required to understand the why the performance difference between CIP tailings and TUF
Fig. 5 Cake moisture versus cycle time with addition of vibration roller assemblies

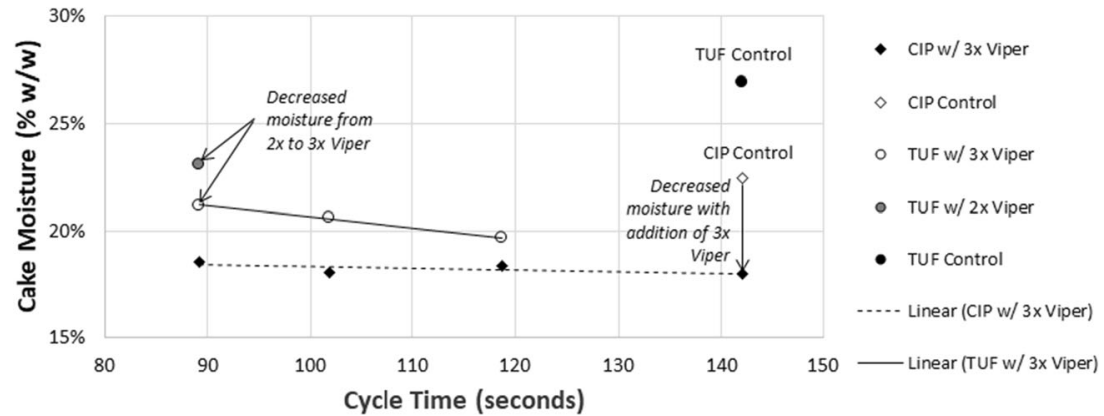


Fig. 6 Throughput increase versus cycle time with addition of vibration roller assemblies

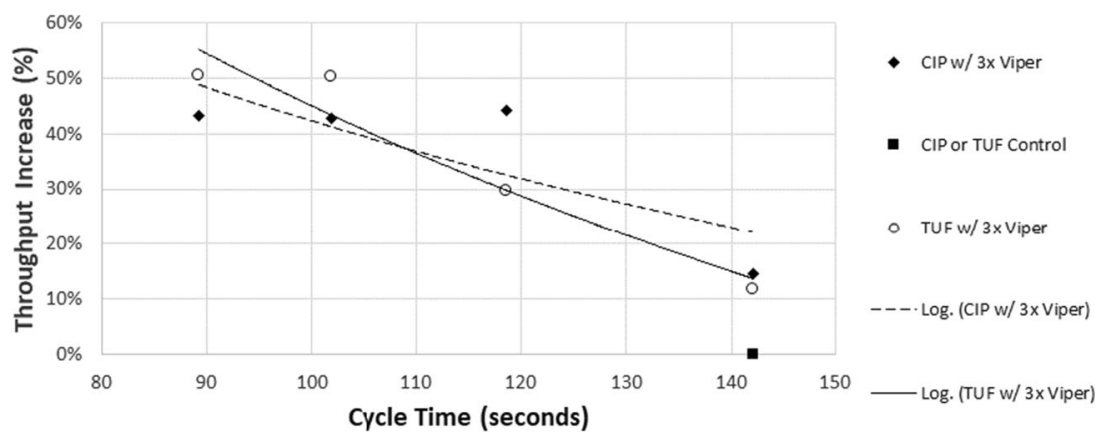

tailings exists to such an extent given the similarity of their respective PSD.

As demonstrated, the cake moisture content increased with increased belt speed (shown in Fig. 5 as cycle time); however, the gradient of the moisture increase versus belt speed trend is relatively flat, particularly for the CIP tailings $(0.6 \% \mathrm{w} / \mathrm{w}$ moisture increase with $53 \mathrm{~s}$ reduction in cycle time). This indicates the lack of time dependence for dewatering via the vibration roller assemblies, which is not the case for reducing cake moisture via conventional "drying." This is further evidenced by the data point in Fig. 5 taken when operating with only two vibration roller assemblies (rather than three), which had a significantly higher moisture content despite all else being equivalent.

This outcome indicates that increased throughput can be achieved by using the vibration roller assemblies to reduce the overall filter cycle time. This increased throughput is quantified in Fig. 6.

Throughput increases in the order of 10-50\% compared to the control were measured. For the CIP tailings sample, these throughput increases were achieved in combination with a cake moisture content $<20 \% \mathrm{w} / \mathrm{w}$. For the TUF sample, throughput increase at the top end of the range resulted in cake moisture $>20 \% \mathrm{w} / \mathrm{w}(20-22 \% \mathrm{w} / \mathrm{w}$ for increases of $\sim 50 \%)$.
A summary of raw results is provided below in Table 1 .

\section{Full-Scale Design}

The pilot testing program facilitated measurement of operating and design data, which forms the basis of a full-scale system design and resultant equipment supply for three vibration roller assemblies to be retro-fitted on the existing vacuum belt filter. The 3D model screenshot in Fig. 7 illustrates the three green Viper Filtration modules near the discharge end of the vacuum belt filter. Figure 8 shows the 3D model of a single Viper Filtration module.

The vibrating action of these rollers improves not only the moisture content but also the production rate and the discharge performance of the cake, particularly for thin cakes. The Viper Filtration technology is also resilient to variance in the feed characteristics. The control system will adapt the settings of the system in response to changes in the process to maintain a consistent output. Elements of Viper Filtration that deliver these positive outcomes include:

i. Substitute the "drying" zone of the filter with more efficient mechanical dewatering using the vibration roller

Table 1 Summary of raw test data

\begin{tabular}{lllllll}
\hline Material & $\begin{array}{l}\text { Cycle time } \\
\text { (seconds) }\end{array}$ & $\begin{array}{l}\text { Cake moisture } \\
(\% \mathrm{w} / \mathrm{w})\end{array}$ & $\begin{array}{l}\text { Equipment } \\
\text { configuration }\end{array}$ & $\begin{array}{l}\text { As tested throughput } \\
\text { increase vs. control (\%) }\end{array}$ & $\begin{array}{l}\text { Washing allowance throughput } \\
\text { increase vs. control }(\%)\end{array}$ & $\begin{array}{l}\text { Moisture reduction vs. } \\
\text { control (\%w/w) }\end{array}$ \\
\hline CIP & 142 & 22.5 & Control & 0 & 0 & 0 \\
& 142 & 18.0 & $3 \times$ Viper & 15 & 15 & 4.5 \\
& 119 & 18.4 & $3 \times$ Viper & 63 & 44 & 4.1 \\
& 102 & 18.1 & $3 \times$ Viper & 59 & 43 & 4.4 \\
TUF & 18.6 & $3 \times$ Viper & 62 & 43 & 3.9 \\
& 142 & 26.9 & Control & 0 & 0 & 0 \\
& 142 & 23.4 & $3 \times$ Viper & 12 & 30 & 3.5 \\
& 119 & 19.7 & $3 \times$ Viper & 33 & 50 & 7.2 \\
& 102 & 20.6 & $3 \times$ Viper & 68 & 50 & 6.3 \\
& 89 & 21.2 & $3 \times$ Viper & 70 & 43 & 5.7 \\
\hline
\end{tabular}


Fig. 7 3D model of the Viper Filtration modules installed on the vacuum belt filter

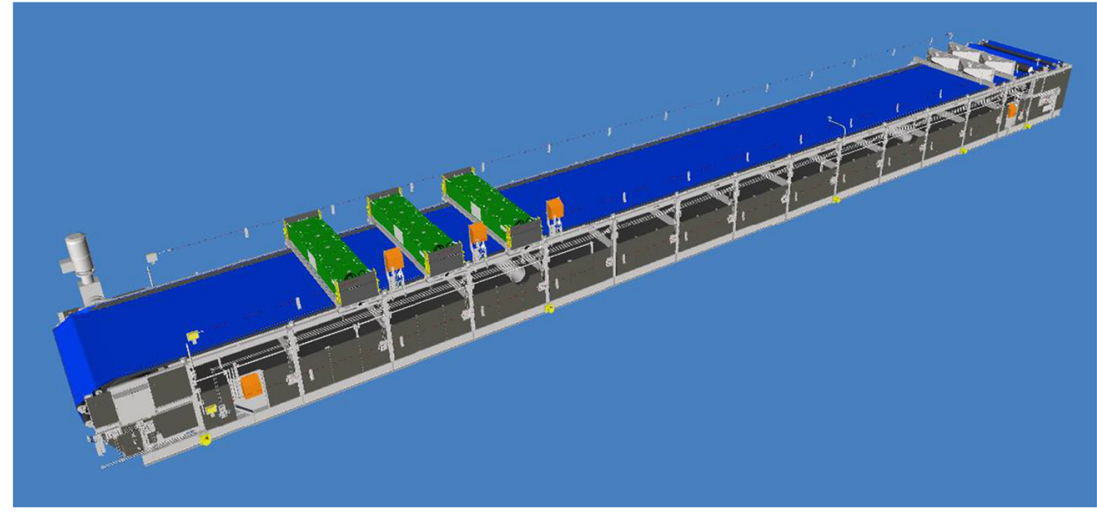

assemblies. Less filter area is required to "dry" the filter cake, making more of the filter's area available for "forming".

ii. Operating with thinner cake optimizes the "form" time, increasing throughput with faster belt speed - the thinner the cake the more efficient the "forming" - the Viper can operate with thin cake as the cake discharge performance is optimized by the boundary layer formed at the cake/ cloth interface.

iii. Maximizing the available vacuum pressure through compaction of the interstitial voids further optimizes the "form": (a) with more of the filter area used for forming and (b) mechanical "drying" of the cake removing the porosity of the cake - the vacuum pressure loss via high airflow are minimized.

iv. The performance of the mechanical dewatering is shown to be more efficient than conventional "drying" delivering the reduced cake moisture.

The technology can be easily scaled up to achieve increased throughput. For instance, considering dewatering of the CIP tailings, multiplying the above system by eight (i.e., 8 belt filters, each with 3 vibration units) is capable of achieving a throughput of 50,000 tpd at $\sim 18 \% \mathrm{w} / \mathrm{w}$ moisture content. Using data from a recent similar project which includes eight belt filters with vibration units (see Fig. 9), the operating cost of the equipment for consumables including filter cloths is approximately $\$ 0.10(\mathrm{AUD}) /$ tonne and the installed power requirement was $4 \mathrm{MW}$.

\section{Conclusions}

The testing program in this case study provided the justification to proceed to full-scale site implementation. The three Viper Filtration modules have been delivered and retrofitted to the existing conventional vacuum belt filter. More broadly, this technology has beneficial industry implications, the most significant being the opportunity to utilize Viper Filtration technology to effectively dewater fine-grained mineral processing tailings at an increased

Fig. 8 3D model of a Viper Filtration module

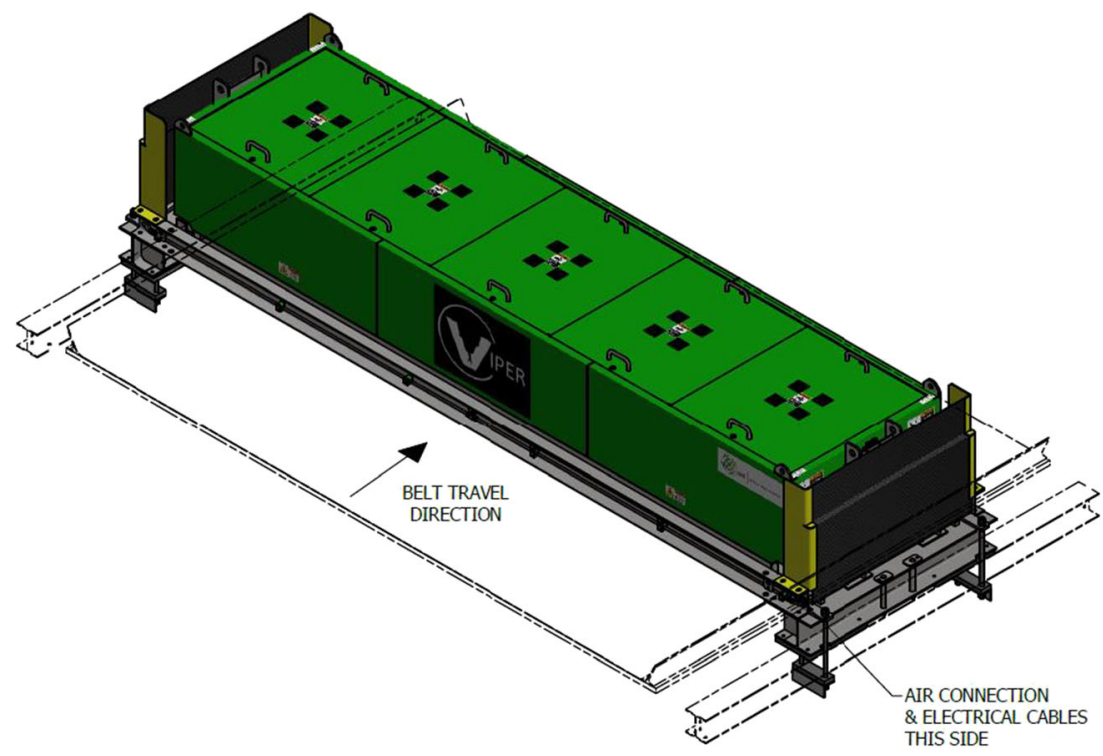


Fig. 9 Layout of 8 off $158 \mathrm{~m}^{2}$ vacuum belt filters equipped with vibratory Viper technology

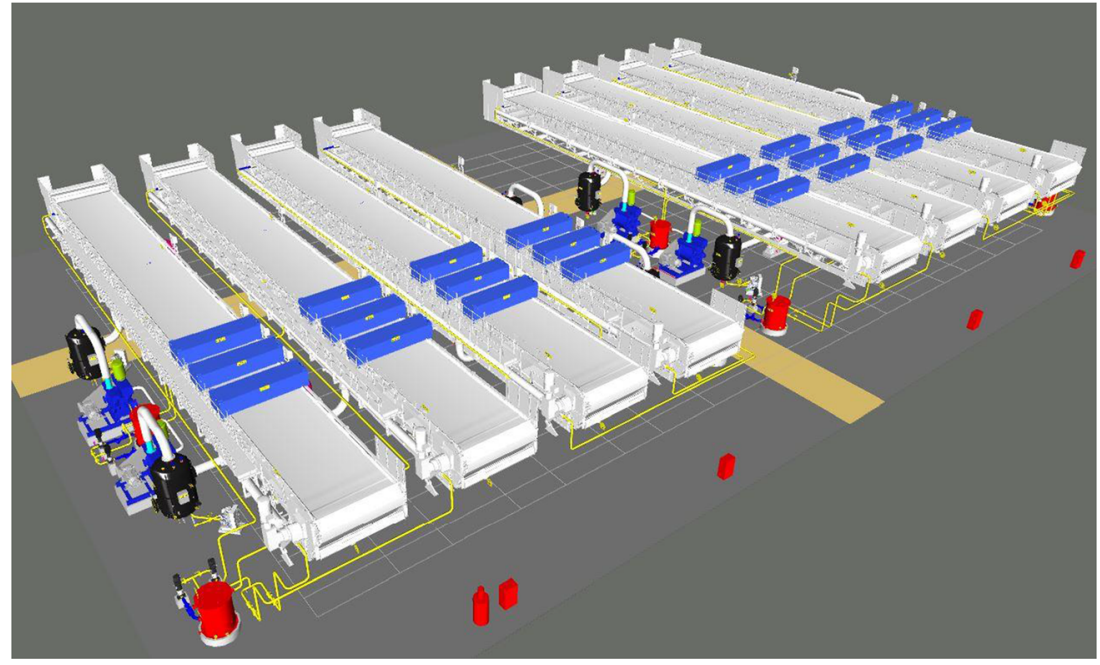

scale. This technology is one of many to answer the call from the MEND [1] Program for the industry to improve technology in support of improved and safer tailings management. The project identified herein is for improved dewatering of tailings for use in paste backfill; however, the technology is also directly translatable for use in dewatering tailings for surface disposal or concentrate filtration applications.

Large, multiple vacuum belt filter installations are not uncommon in the minerals processing industry as they meet the needs of the modern user, including:

i. Simple, scalable technology with more than 25 years of operational history in the industry

ii. Continuous processing machine with high availability (> $90 \%)$

iii. Operates with a single filter cloth (typical lifetime 612 months)

iv. Low supporting infrastructure costs and complexity

v. Cake discharge from multiple machines can be directed to an overland conveyor

The technology discussed in this paper delivers these features that can be used to dewater large-scale mineral tailings potentially suitable for surface disposal ("dry stack"), co-disposal, or paste backfill as well as concentrate applications.

\section{Compliance with Ethical Standards}

Conflict of Interest Oliver Whatnall is an employee of Jord International Pty Ltd who has a commercial interest in the subject of this manuscript and co-inventor of patent in the subject of this manuscript. Kevin Barber is an employee of Jord International Pty Ltd who has a commercial interest in the subject of this manuscript and co-inventor of patent in the subject of this manuscript. Dr. Peter Robinson is a researcher at The University of Newcastle, who has a commercial interest in the subject of this manuscript and co-inventor of patent in the subject of this manuscript.

Open Access This article is licensed under a Creative Commons Attribution 4.0 International License, which permits use, sharing, adaptation, distribution and reproduction in any medium or format, as long as you give appropriate credit to the original author(s) and the source, provide a link to the Creative Commons licence, and indicate if changes were made. The images or other third party material in this article are included in the article's Creative Commons licence, unless indicated otherwise in a credit line to the material. If material is not included in the article's Creative Commons licence and your intended use is not permitted by statutory regulation or exceeds the permitted use, you will need to obtain permission directly from the copyright holder. To view a copy of this licence, visit http://creativecommons.org/licenses/by/4.0/.

\section{Reference}

1. Mine Environment Neutral Drainage (MEND) Program (2017) "Study of tailings management technologies." MEND Report 2501 October 2017. http://mend-nedem.org/wp-content/uploads/2.50. 1Tailings_Management_TechnologiesL.pdf

Publisher's Note Springer Nature remains neutral with regard to jurisdictional claims in published maps and institutional affiliations. 